\title{
ENAH Protein Isoform Expression Assay
}

National Cancer Institute

\section{Source}

National Cancer Institute. ENAH Protein Isoform Expression Assay. NCI Thesaurus. Code C120021.

A proprietary immunofluorescent assay that quantifies the relative expression levels of all isoforms of the actin regulatory protein, protein enabled homolog (Mena or ENAH), including Mena(INV) and Mena11a. Mena(INV) expression is elevated and Mena11a expression is diminished or absent in invasive tumor cells, which may have increased metastatic potential. Therefore, this assay may be able to predict a patient's risk for tumor metastasis. 